\title{
Utilization of a Stress Ball to Diminish Anxiety During Nail Surgery
}

\author{
Jose W. Ricardo, MD; Shari R. Lipner, MD, PhD
}

Anxiety is commonly encountered by patients undergoing nail surgery. Squeezing a stress ball with the free hand does not interfere with the surgical procedure, and it is a widely accessible and cost-effective technique that reduces anxiety in patients undergoing nail surgery.

Cutis. 2020;105:294.

\section{Practice Gap}

Anxiety is common in patients undergoing surgery with general anesthesia and may be exacerbated in patients undergoing dermatologic surgery with local anesthesia. Apprehension might be worse for nail surgery patients because the nail unit is highly innervated and vascular. Many patients fear the anesthetic injections, and there often is pain postoperatively. Perioperative anxiety correlates with increased postoperative pain, ${ }^{1}$ analgesic use, ${ }^{2}$ and delayed recovery. ${ }^{3}$ Several alternatives have been proposed to decrease perioperative anxiety, including nonpharmacologic interventions such as using educational videos, personalized music, hand holding, art activities, and virtual reality, as well as pharmacologic interventions such as benzodiazepines. However, these techniques have not been well studied for nail surgery.

\section{The Technique}

Patients generally are anxious about nail surgery secondary to the pain associated with the local anesthetic infiltration; hence, it is crucial to decrease anxiety during this initial step. In our practice, we provide patients with a palmsized stress ball made of closed-cell polyurethane foam rubber before surgery. Patients are then instructed to hold the stress ball with the free hand and squeeze it whenever they feel anxious or when they feel any discomfort related to the procedure (Figure). A variety of balls can be bought for less than $\$ 1$ each, thus making it a cost-effective option.

\section{Practice Implications}

Holding a stress ball has been found to reduce both pain and anxiety in patients undergoing conscious surgery. ${ }^{4}$ Furthermore, squeezing a stress ball perioperatively may increase feelings of empowerment, given that patients have

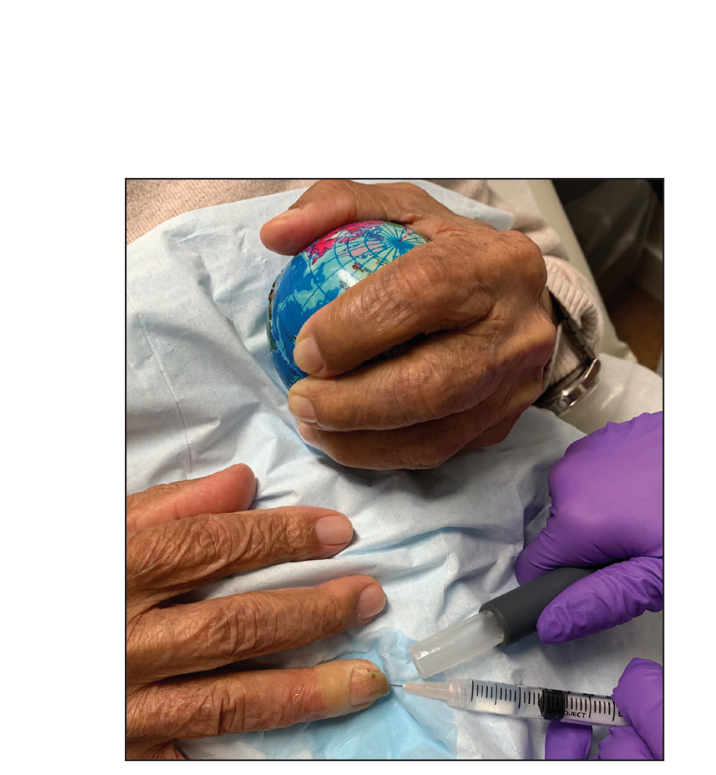

A patient holding a stress ball with the free hand while the nail surgeon is infiltrating the affected nail unit with the local anesthetic agent.

direct control over the object, which in turn may have a positive effect on anxiety and patient satisfaction without interfering with the surgical procedure. ${ }^{5}$ Holding a stress ball is a safe, widely accessible, and inexpensive technique that may aid in decreasing patients' anxiety related to nail surgery. Nonetheless, controlled clinical trials assessing the efficacy of this method in reducing anxiety related to nail surgery are needed to determine its benefit compared to other methods.

\section{REFERENCES}

1. Carr EC, Nicky Thomas V, Wilson-Barnet J. Patient experiences of anxiety, depression and acute pain after surgery: a longitudinal perspective. Int J Nurs Stud. 2005;42:521-530.

2. Powell R, Johnston M, Smith WC, et al. Psychological risk factors for chronic post-surgical pain after inguinal hernia repair surgery: a prospective cohort study. Eur J Pain. 2012;16:600-610.

3. Mavros MN, Athanasiou S, Gkegkes ID, et al. Do psychological variables affect early surgical recovery? PLoS One. 2011;6:e20306.

4. Hudson BF, Ogden J, Whiteley MS. Randomized controlled trial to compare the effect of simple distraction interventions on pain and anxiety experienced during conscious surgery. Eur J Pain. 2015;19:1447-1455.

5. Foy CR, Timmins F. Improving communication in day surgery settings. Nurs Stand. 2004;19:37-42.

From the Department of Dermatology, Weill Cornell Medicine, New York, New York.

The authors report no conflict of interest.

Correspondence: Shari R. Lipner, MD, PhD, 1305 York Ave, New York, NY 10021 (sh19032@med.cornell.edu). 